\title{
THE EFFECT OF CLIMAX AND ANTICLIMAX ORDER OF PRESENTATION ON MEMORY
}

\author{
By Henry Foster Adams, University of Michigan
}

In previous papers, ${ }^{1}$ the memory efficiency of size of advertisements, of their frequency of presentation with both duplication and variation, and of mixed sizes of advertisements, have been discussed. In the last paper mentioned, it was found that the memory value of the same total amount of space varied as it was made up of different combinations of sizes. For instance a series made up of 3 half and 1 quarter page advertisements of the same commodity was found to have a greater memorability than a similar series composed of 1 full and 3 quarter pages, yet the total area is the same in both cases, 1.75 pages, and the number of presentations is 4 in each case.

But a question which was left unsolved in that experiment was whether the order in which the mixed sizes were presented to the subject had any effect upon the memorability of the series. For example, if a firm advertises 4 times with mixed sizes of advertisements, is it more effective upon memory to present the larger sized advertisements at the beginning or end of the series.

To test the point 3 dummies were prepared, as follows. In dummy $A$, for instance, each firm advertised 4 times, the different insertions being variations and at least two sizes of advertisements being included in the 4 insertions. Each different arrangement of sizes was likewise represented by four different firms. With two of the firms, the larger sizes appeared first, followed by the smaller ones and with the other two firms for the same combination of sizes, the advertisements of smaller area appeared first, followed by the larger ones. In all, the following combinations were presented:-

1 The Relative Importance of Size and Frequency in Forming Associations, J. of Phil., Psy., and Sci. Meth., Vol. XII, 1915, pp. 477-491.

The Relative Memory and Values of Duplication and Variation in Advertising, ibid., Vol. XIII, 1916, pp. 141-152.

The Memory of Mixed Sizes of Advertisements, J. of Exper. Psychol. Vol. II, 1917, pp. 448-465. 


\begin{tabular}{llll} 
Dummy & A & B & C \\
\hline FFFH & 2 & 2 & 2 \\
HFFF & 2 & 2 & 2 \\
FFFQ & 2 & 2 & 2 \\
QFFF & 2 & 2 & 2 \\
FFHH & 2 & 2 & 2 \\
HHFF & 2 & 2 & 2 \\
FFQQ & 2 & 2 & 2 \\
QQFF & 2 & 2 & 2 \\
FFHQ & 2 & 2 & 2 \\
QHFF & 2 & 2 & 2 \\
FHHH & 2 & 2 & 2 \\
HHHF & 2 & 2 & 2 \\
FQQQ & 2 & 2 & 2 \\
QOQF & 2 & 2 & 2 \\
FHHQ & 2 & 2 & 2 \\
QHHF & 2 & 2 & 2 \\
FHQQ & 2 & 2 & 2 \\
QQHF & 2 & 2 & 2 \\
HHHO & 2 & 2 & 2 \\
QHHH & 2 & 2 & 2 \\
HHQQ & 2 & 2 & 2 \\
QQHH & 2 & 2 & 2 \\
HQQQ & 2 & 2 & 2 \\
QQQH & 2 & 2 & 2 \\
\hline
\end{tabular}

In the experiment, part of the subjects began reading the dummy at page 1 and went along progressively to the end; others began at the end and read back to page 1 . This was easily accomplished by turning the dummy upside down. Therefore, to those subjects who read the dummy through in the forward direction, the advertisements of a certain firm would be seen in the order FFFH; while to those who read it through in the reverse direction, the same advertisements of the same firm would appear in the order HFFF. In this way each series of advertisements was seen by the subjects in both the climax and anti-climax order. For the sake of convenience of expression, the series of advertisements is said to be in the climax order if the smaller spaces appear first followed by the larger ones; in the anti-climax order if the larger spaces are seen first followed by the smaller ones.

With dummy A, 93 subjects were used in the direct or forward order of reading, 68 in the reverse order; in dummy B 95 in the direct order, 58 in the reverse; in dummy C 79 in the forward order and 70 in the reverse order. This makes a total of 463 subjects. 
Since the results of the experiments may be considered in several ways and the conclusions to be drawn from the experiment depend in part upon the method of treatment, the original data will be presented. It is given in Table I.

TABLE I

\begin{tabular}{|c|c|c|c|c|c|c|c|c|c|c|c|c|}
\hline \multirow{2}{*}{$\begin{array}{c}\text { Order } \\
\text { of Ads. }\end{array}$} & \multicolumn{4}{|c|}{ Dummy $A$} & \multicolumn{4}{|c|}{ Dummy $\mathbf{B}$} & \multicolumn{4}{|c|}{ Dummy C } \\
\hline & $\mathbf{D}$ & $\mathbf{R}$ & & $\mathrm{Cl}_{\mathbf{l}}$ & D & $\mathbf{R}$ & Ant & $\mathrm{Cl}$ & $\mathbf{D}$ & & & Clı \\
\hline \multirow{3}{*}{$\begin{array}{l}\text { FFFH } \ldots \ldots \\
\text { HFFF } \ldots \ldots\end{array}$} & 137 & 103 & 137 & 103 & 53 & 29 & 53 & 29 & 92 & 76 & 92 & 76 \\
\hline & $\begin{array}{l}70 \\
70\end{array}$ & $\begin{array}{l}\mathbf{0 4} \\
\mathbf{6 5} \\
53\end{array}$ & $\begin{array}{l}65 \\
65 \\
53\end{array}$ & $\begin{array}{l}\mathbf{4} \\
70 \\
70\end{array}$ & $\begin{array}{l}40 \\
49 \\
58\end{array}$ & $\begin{array}{l}21 \\
30 \\
34\end{array}$ & $\begin{array}{l}40 \\
30 \\
34\end{array}$ & $\begin{array}{l}41 \\
49 \\
58\end{array}$ & $\begin{array}{l}72 \\
72 \\
69\end{array}$ & $\begin{array}{l}71 \\
92\end{array}$ & $\begin{array}{l}41 \\
71 \\
92\end{array}$ & $\begin{array}{l}72 \\
72 \\
69\end{array}$ \\
\hline & & & 333 & 307 & & & 165 & 157 & & & 303 & 269 \\
\hline FFFQ $\ldots \ldots$ & 74 & 56 & 74 & 56 & 49 & 26 & 49 & 26 & 76 & 81 & 76 & 81 \\
\hline \multirow[t]{2}{*}{ QFFF... } & 51 & $\begin{array}{l}82 \\
86 \\
37\end{array}$ & $\begin{array}{r}121 \\
86 \\
37\end{array}$ & $\begin{array}{r}84 \\
104 \\
51\end{array}$ & $\begin{array}{l}38 \\
36 \\
53\end{array}$ & $\begin{array}{l}18 \\
11 \\
28\end{array}$ & $\begin{array}{l}38 \\
11 \\
28\end{array}$ & $\begin{array}{l}18 \\
36 \\
53\end{array}$ & $\begin{array}{l}84 \\
32 \\
52\end{array}$ & $\begin{array}{l}71 \\
34 \\
49\end{array}$ & $\begin{array}{l}84 \\
34 \\
49\end{array}$ & $\begin{array}{l}71 \\
32 \\
52\end{array}$ \\
\hline & & & 318 & 293 & & & 126 & 133 & & & 243 & 236 \\
\hline \multirow{3}{*}{$\begin{array}{l}\text { FFHH... . } \\
\text { HHFF . . . . }\end{array}$} & 55 & 42 & 55 & 42 & 44 & 22 & 44 & 22 & 66 & 81 & 66 & 81 \\
\hline & $\begin{array}{r}91 \\
110\end{array}$ & $\begin{array}{l}66 \\
91\end{array}$ & $\begin{array}{l}66 \\
91\end{array}$ & $\begin{array}{r}91 \\
110\end{array}$ & $\begin{array}{l}83 \\
23\end{array}$ & $\begin{array}{l}50 \\
11\end{array}$ & $\begin{array}{l}50 \\
50 \\
11\end{array}$ & $\begin{array}{l}83 \\
83\end{array}$ & $\begin{array}{l}12 \\
87\end{array}$ & $\begin{array}{l}37 \\
89\end{array}$ & $\begin{array}{l}37 \\
89\end{array}$ & $\begin{array}{l}32 \\
87\end{array}$ \\
\hline & & & 283 & 305 & & & 155 & 150 & & & 209 & 225 \\
\hline FFQQ $\ldots . .$. & 23 & 22 & 23 & 22 & 57 & 15 & 57 & 15 & 87 & 101 & 87 & 101 \\
\hline \multirow[t]{2}{*}{ QQFF.. } & $\begin{array}{r}28 \\
32\end{array}$ & $\begin{array}{l}39 \\
39 \\
38\end{array}$ & $\begin{array}{r}115 \\
39 \\
38\end{array}$ & $\begin{array}{l}0 \\
28 \\
32\end{array}$ & $\begin{array}{r}3 \\
33 \\
100\end{array}$ & $\begin{array}{l}29 \\
61\end{array}$ & 22 & $\begin{array}{r}33 \\
100\end{array}$ & $\begin{array}{r}107 \\
35\end{array}$ & $\begin{array}{r}113 \\
47\end{array}$ & $\begin{array}{r}113 \\
47\end{array}$ & $\begin{array}{r}81 \\
107 \\
35\end{array}$ \\
\hline & & & 215 & 169 & & & 197 & 177 & & & 342 & 324 \\
\hline \multirow{3}{*}{$\begin{array}{l}\text { FFHQ } \ldots \ldots \\
\text { QHFF. } \ldots \ldots\end{array}$} & 77 & 51 & 77 & 51 & 27 & 5 & 27 & 5 & 22 & 22 & 22 & 22 \\
\hline & $\begin{array}{l}76 \\
36\end{array}$ & $\begin{array}{l}61 \\
30\end{array}$ & $\begin{array}{l}61 \\
30\end{array}$ & $\begin{array}{l}76 \\
36\end{array}$ & $\begin{array}{l}30 \\
37\end{array}$ & $\begin{array}{l}15 \\
17\end{array}$ & $\begin{array}{l}15 \\
17\end{array}$ & $\begin{array}{l}30 \\
37\end{array}$ & $\begin{array}{l}89 \\
55\end{array}$ & $\begin{array}{l}95 \\
67\end{array}$ & $\begin{array}{l}95 \\
67\end{array}$ & $\begin{array}{l}89 \\
55\end{array}$ \\
\hline & & & 225 & 208 & & & 173 & 126 & & & 220 & 223 \\
\hline \multirow{3}{*}{$\begin{array}{l}\text { FHHН, . . . } \\
\text { HНHF, . . }\end{array}$} & 60 & 41 & 60 & 41 & 59 & 26 & 59 & 26 & 18 & 27 & 18 & 27 \\
\hline & $\begin{array}{l}88 \\
60\end{array}$ & $\begin{array}{l}57 \\
63\end{array}$ & $\begin{array}{l}57 \\
63\end{array}$ & $\begin{array}{l}88 \\
60\end{array}$ & $\begin{array}{l}18 \\
55\end{array}$ & $\begin{array}{r}7 \\
27\end{array}$ & $\begin{array}{r}7 \\
27\end{array}$ & 18 & $\begin{array}{r}106 \\
61\end{array}$ & 91 & $\begin{array}{l}91 \\
53\end{array}$ & $\begin{array}{r}106 \\
61\end{array}$ \\
\hline & & & 245 & 225 & & & 194 & 160 & & & 280 & 304 \\
\hline \multirow{3}{*}{$\begin{array}{l}\text { FQQQ..... } \\
\text { QQQF... . . }\end{array}$} & 70 & 68 & 70 & 68 & 65 & 30 & 65 & 30 & 25 & 23 & 25 & 23 \\
\hline & $\begin{array}{l}24 \\
81 \\
50\end{array}$ & $\begin{array}{l}10 \\
28 \\
36\end{array}$ & $\begin{array}{l}24 \\
28 \\
36\end{array}$ & $\begin{array}{l}10 \\
31 \\
50\end{array}$ & $\begin{array}{l}45 \\
74 \\
52\end{array}$ & $\begin{array}{l}10 \\
43 \\
21\end{array}$ & $\begin{array}{l}45 \\
43 \\
21\end{array}$ & $\begin{array}{l}19 \\
74 \\
52\end{array}$ & $\begin{array}{l}59 \\
44 \\
96\end{array}$ & $\begin{array}{l}01 \\
65 \\
87\end{array}$ & $\begin{array}{l}29 \\
65 \\
87\end{array}$ & $\begin{array}{l}61 \\
44 \\
96\end{array}$ \\
\hline & & & 155 & 159 & & & 174 & 175 & & & 236 & 224 \\
\hline \multirow[t]{2}{*}{$\begin{array}{l}\text { FHHQ ........ } \\
\text { QHHF ....... }\end{array}$} & $\begin{array}{r}27 \\
33 \\
50 \\
9\end{array}$ & $\begin{array}{l}26 \\
26 \\
49 \\
12\end{array}$ & $\begin{array}{l}27 \\
33 \\
49 \\
12\end{array}$ & $\begin{array}{l}26 \\
26 \\
50 \\
9\end{array}$ & $\begin{array}{r}24 \\
105 \\
63 \\
13\end{array}$ & $\begin{array}{r}10 \\
43 \\
34 \\
8\end{array}$ & $\begin{array}{r}24 \\
105 \\
34 \\
8\end{array}$ & $\begin{array}{l}10 \\
43 \\
63 \\
13\end{array}$ & $\begin{array}{r}59 \\
41 \\
104 \\
35\end{array}$ & $\begin{array}{l}62 \\
50 \\
83 \\
46\end{array}$ & $\begin{array}{l}59 \\
41 \\
83 \\
46\end{array}$ & $\begin{array}{r}62 \\
50 \\
104 \\
35\end{array}$ \\
\hline & & & 121 & 111 & & & 171 & 129 & & & 229 & 251 \\
\hline \multirow{3}{*}{$\begin{array}{l}\text { FHQQ... .. . } \\
\text { QQHF. ... . }\end{array}$} & 42 & 38 & 42 & 38 & 37 & 21 & 37 & 21 & 77 & 78 & 77 & 78 \\
\hline & $\begin{array}{l}91 \\
35\end{array}$ & $\begin{array}{l}84 \\
40\end{array}$ & $\begin{array}{l}84 \\
40\end{array}$ & $\begin{array}{l}91 \\
35\end{array}$ & $\begin{array}{l}29 \\
66\end{array}$ & $\begin{array}{l}17 \\
28\end{array}$ & $\begin{array}{l}17 \\
28\end{array}$ & $\begin{array}{l}29 \\
66\end{array}$ & 52 & $\begin{array}{l}70 \\
15\end{array}$ & $\begin{array}{l}70 \\
15\end{array}$ & $\begin{array}{l}52 \\
12\end{array}$ \\
\hline & & & 226 & 204 & & & 179 & 164 & & & 254 & 230 \\
\hline
\end{tabular}


TABLE I-Continued

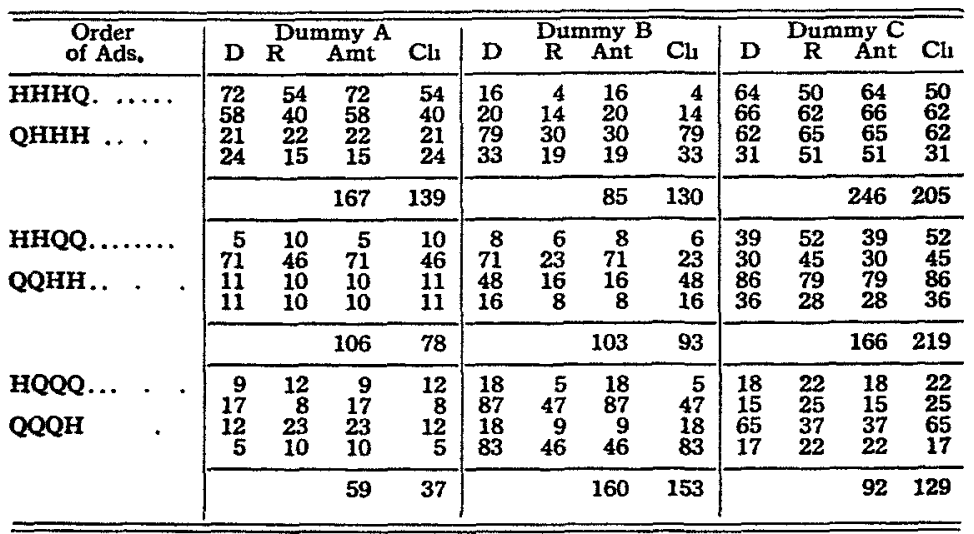

The column of initials at the left gives the order of presentation of the advertisements, $F$ standing for full page, $H$ for half page and $Q$ for quarter page. The second, labeled $D$, gives the gross credits received by the various series of advertisements when the subjects read in the forward or direct order, starting at page 1 . The third column, labeled $\mathrm{R}$ gives the gross credits received by the advertisements when the dummy was read through in the reverse direction. The fourth column, headed by Ant. gives the credits for the advertisements which were read through in the anti-climax order; and in the fifth column, headed Cli. the credits for those advertisements which were read through in the climax order. The other columns gives the same data for dummies $\mathrm{B}$ and $\mathrm{C}$.

TABLE II

\begin{tabular}{|c|c|c|c|c|c|c|c|c|}
\hline \multirow[b]{2}{*}{ 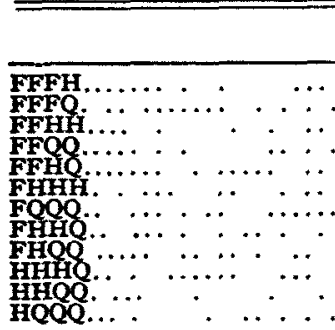 } & \multicolumn{2}{|c|}{$\underset{\text { Anti }}{\text { Dummy A }}$} & \multicolumn{2}{|c|}{$\begin{array}{l}\text { Dummy } \mathrm{B} \\
\text { Antı } \mathrm{Cl}^{-}\end{array}$} & \multicolumn{2}{|c|}{$\underset{\text { Ant } 1}{\text { Dummy C }}$} & \multicolumn{2}{|c|}{ Total } \\
\hline & $\begin{array}{r}333 \\
318 \\
283 \\
215 \\
225 \\
245 \\
155 \\
121 \\
226 \\
167 \\
106 \\
59\end{array}$ & $\begin{array}{r}307 \\
293 \\
305 \\
169 \\
208 \\
225 \\
159 \\
111 \\
204 \\
139 \\
78 \\
37\end{array}$ & $\begin{array}{l}165 \\
126 \\
155 \\
197 \\
173 \\
194 \\
174 \\
171 \\
179 \\
85 \\
103 \\
160\end{array}$ & $\begin{array}{r}157 \\
133 \\
150 \\
177 \\
126 \\
160 \\
175 \\
129 \\
164 \\
130 \\
93 \\
153\end{array}$ & $\begin{array}{r}303 \\
243 \\
209 \\
342 \\
220 \\
280 \\
236 \\
229 \\
254 \\
246 \\
166 \\
92\end{array}$ & $\begin{array}{l}269 \\
236 \\
225 \\
324 \\
223 \\
304 \\
224 \\
251 \\
230 \\
205 \\
219 \\
129\end{array}$ & $\begin{array}{l}801 \\
687 \\
647 \\
754 \\
618 \\
719 \\
565 \\
521 \\
659 \\
498 \\
375 \\
311\end{array}$ & $\begin{array}{l}733 \\
662 \\
680 \\
670 \\
557 \\
689 \\
558 \\
491 \\
598 \\
474 \\
390 \\
\mathbf{3 1 9}\end{array}$ \\
\hline & 0 & 235 & 1882 & 1747 & 2820 & 2839 & $\begin{array}{r}7155 \\
105\end{array}$ & $\begin{array}{r}6821 \\
100\end{array}$ \\
\hline
\end{tabular}

In Table II, a summary of the climax and anti-climax effects 
is given, indicating that the anti-climax order of presentation is somewhat more effective as far as memory is concerned.

But it must be remembered that these are gross figures and furthermore that unequal numbers of subjects were used in the forward and backward turning of the pages of the dummy. To compensate for this the credits for each advertisement were put into percentages which were multiplied

TABLE III

\begin{tabular}{|c|c|c|c|c|c|c|c|c|c|c|c|c|}
\hline $\begin{array}{l}\text { Order } \\
\text { of Ads. }\end{array}$ & \multicolumn{4}{|c|}{$A$} & \multicolumn{4}{|c|}{$\bar{B}$} & D & $\mathrm{R}$ & Ant & $\mathrm{Cl}_{\mathbf{i}}$ \\
\hline \multirow[t]{2}{*}{$\begin{array}{l}\text { FFFH } \\
\text { HFFF. }\end{array}$} & $\begin{array}{l}529 \\
302 \\
270 \\
\mathbf{2 7 0}\end{array}$ & $\begin{array}{l}494 \\
306 \\
312 \\
254\end{array}$ & $\begin{array}{l}529 \\
302 \\
312 \\
\mathbf{2 5 4}\end{array}$ & $\begin{array}{l}494 \\
306 \\
270 \\
270\end{array}$ & $\begin{array}{l}218 \\
198 \\
201 \\
238\end{array}$ & $\begin{array}{l}244 \\
176 \\
252 \\
286\end{array}$ & $\begin{array}{l}218 \\
198 \\
252 \\
286\end{array}$ & $\begin{array}{l}244 \\
176 \\
201 \\
238\end{array}$ & $\begin{array}{l}332 \\
174 \\
259 \\
248\end{array}$ & $\begin{array}{l}262 \\
179 \\
245 \\
318\end{array}$ & $\begin{array}{l}332 \\
174 \\
245 \\
318\end{array}$ & $\begin{array}{l}262 \\
179 \\
259 \\
248\end{array}$ \\
\hline & & & 1397 & 1340 & & & 954 & 859 & & & 1069 & 948 \\
\hline \multirow[t]{2}{*}{ FFFQ. $\ldots \ldots$} & $\begin{array}{l}286 \\
466 \\
402 \\
198\end{array}$ & $\begin{array}{l}268 \\
393 \\
412 \\
177\end{array}$ & $\begin{array}{l}286 \\
466 \\
412 \\
177\end{array}$ & $\begin{array}{l}268 \\
393 \\
402 \\
198\end{array}$ & $\begin{array}{l}202 \\
156 \\
148 \\
218\end{array}$ & $\begin{array}{l}218 \\
151 \\
92 \\
235\end{array}$ & $\begin{array}{r}202 \\
156 \\
92 \\
235\end{array}$ & $\begin{array}{l}218 \\
151 \\
148 \\
218\end{array}$ & $\begin{array}{l}275 \\
304 \\
108 \\
187\end{array}$ & $\begin{array}{l}280 \\
245 \\
118 \\
170\end{array}$ & $\begin{array}{l}275 \\
304 \\
118 \\
170\end{array}$ & $\begin{array}{l}280 \\
245 \\
108 \\
187\end{array}$ \\
\hline & & & 1341 & 1261 & & & 685 & 735 & & & 867 & 820 \\
\hline \multirow[t]{2}{*}{$\begin{array}{l}\text { FFHH... . } \\
\text { HHFF.... }\end{array}$} & $\begin{array}{l}213 \\
275 \\
352 \\
425\end{array}$ & $\begin{array}{l}201 \\
296 \\
316 \\
435\end{array}$ & $\begin{array}{l}213 \\
275 \\
316 \\
435\end{array}$ & $\begin{array}{l}201 \\
296 \\
352 \\
425\end{array}$ & $\begin{array}{r}181 \\
206 \\
341 \\
94\end{array}$ & $\begin{array}{r}185 \\
185 \\
420 \\
92\end{array}$ & $\begin{array}{r}181 \\
206 \\
420 \\
92\end{array}$ & $\begin{array}{r}185 \\
185 \\
341 \\
94\end{array}$ & $\begin{array}{r}238 \\
61 \\
79 \\
314\end{array}$ & $\begin{array}{l}280 \\
121 \\
128 \\
308\end{array}$ & $\begin{array}{r}238 \\
61 \\
128 \\
308\end{array}$ & $\begin{array}{r}280 \\
121 \\
79 \\
314\end{array}$ \\
\hline & & & 1239 & 1274 & & & 899 & 805 & & & 735 & 794 \\
\hline \multirow[t]{2}{*}{ FFQQ } & $\begin{array}{r}89 \\
445 \\
104 \\
124\end{array}$ & $\begin{array}{l}102 \\
415 \\
187 \\
182\end{array}$ & $\begin{array}{r}89 \\
445 \\
187 \\
182\end{array}$ & $\begin{array}{l}102 \\
415 \\
104 \\
124\end{array}$ & $\begin{array}{l}235 \\
235 \\
135 \\
410\end{array}$ & $\begin{array}{l}126 \\
244 \\
185 \\
512\end{array}$ & $\begin{array}{l}235 \\
235 \\
185 \\
512\end{array}$ & $\begin{array}{l}126 \\
244 \\
135 \\
410\end{array}$ & $\begin{array}{l}314 \\
344 \\
385 \\
126\end{array}$ & $\begin{array}{l}350 \\
280 \\
390 \\
163\end{array}$ & $\begin{array}{l}314 \\
344 \\
390 \\
163\end{array}$ & $\begin{array}{l}350 \\
280 \\
385 \\
126\end{array}$ \\
\hline & & & 903 & 745 & & & 1167 & 915 & & & 1211 & 1141 \\
\hline \multirow[t]{2}{*}{$\begin{array}{l}\text { FFHQ.. } \\
\text { QHFF }\end{array}$} & $\begin{array}{l}298 \\
221 \\
293 \\
149\end{array}$ & $\begin{array}{l}244 \\
215 \\
293 \\
144\end{array}$ & $\begin{array}{l}298 \\
221 \\
293 \\
144\end{array}$ & $\begin{array}{l}244 \\
215 \\
293 \\
149\end{array}$ & $\begin{array}{l}106 \\
469 \\
123 \\
152\end{array}$ & $\begin{array}{r}42 \\
454 \\
126 \\
143\end{array}$ & $\begin{array}{l}106 \\
469 \\
126 \\
143\end{array}$ & $\begin{array}{r}42 \\
454 \\
123 \\
152\end{array}$ & $\begin{array}{r}79 \\
130 \\
320 \\
199\end{array}$ & $\begin{array}{r}76 \\
196 \\
338 \\
232\end{array}$ & $\begin{array}{r}79 \\
130 \\
338 \\
232\end{array}$ & $\begin{array}{r}76 \\
196 \\
320 \\
199\end{array}$ \\
\hline & & & 956 & 901 & & & 844 & 771 & & & 779 & 791 \\
\hline \multirow[t]{2}{*}{$\begin{array}{l}\text { FHHН ... . } \\
\text { HHHF. .. } .\end{array}$} & $\begin{array}{l}232 \\
251 \\
340 \\
\mathbf{2 3 2} \\
\end{array}$ & $\begin{array}{l}198 \\
172 \\
264 \\
302\end{array}$ & $\begin{array}{l}232 \\
251 \\
264 \\
302\end{array}$ & $\begin{array}{l}198 \\
172 \\
340 \\
232\end{array}$ & $\begin{array}{r}243 \\
420 \\
74 \\
226\end{array}$ & $\begin{array}{l}218 \\
511 \\
59 \\
227\end{array}$ & $\begin{array}{r}243 \\
420 \\
59 \\
227\end{array}$ & $\begin{array}{l}218 \\
511 \\
74 \\
226\end{array}$ & $\begin{array}{r}65 \\
426 \\
381 \\
219\end{array}$ & $\begin{array}{r}93 \\
380 \\
315 \\
183\end{array}$ & $\begin{array}{r}65 \\
426 \\
315 \\
183\end{array}$ & $\begin{array}{r}93 \\
380 \\
381 \\
219\end{array}$ \\
\hline & & & 1049 & 942 & & & 949 & 1029 & & & 989 & 1073 \\
\hline \multirow[t]{2}{*}{$\begin{array}{l}\text { FQQQ . ... } \\
\text { QQQF ... }\end{array}$} & $\begin{array}{r}270 \\
93 \\
119 \\
193\end{array}$ & $\begin{array}{r}325 \\
48 \\
134 \\
172\end{array}$ & $\begin{array}{r}270 \\
93 \\
134 \\
172\end{array}$ & $\begin{array}{r}325 \\
48 \\
119 \\
193\end{array}$ & $\begin{array}{l}268 \\
185 \\
304 \\
214\end{array}$ & $\begin{array}{l}251 \\
159 \\
362 \\
177\end{array}$ & $\begin{array}{l}268 \\
185 \\
362 \\
177\end{array}$ & $\begin{array}{l}251 \\
159 \\
304 \\
214\end{array}$ & $\begin{array}{r}90 \\
214 \\
158 \\
345\end{array}$ & $\begin{array}{r}80 \\
210 \\
224 \\
301\end{array}$ & $\begin{array}{r}90 \\
214 \\
224 \\
301\end{array}$ & $\begin{array}{r}80 \\
210 \\
158 \\
345\end{array}$ \\
\hline & & & 669 & 685 & & & 992 & 928 & & & 829 & 793 \\
\hline \multirow[t]{2}{*}{$\begin{array}{l}\text { FHHQ... } \ldots \ldots \\
\text { QHHF } \ldots \ldots\end{array}$} & $\begin{array}{r}102 \\
128 \\
193 \\
35\end{array}$ & $\begin{array}{r}124 \\
124 \\
234 \\
58\end{array}$ & $\begin{array}{r}102 \\
128 \\
234 \\
58\end{array}$ & $\begin{array}{r}124 \\
124 \\
193 \\
35\end{array}$ & $\begin{array}{r}99 \\
431 \\
259 \\
53\end{array}$ & $\begin{array}{r}84 \\
362 \\
285 \\
67\end{array}$ & $\begin{array}{r}99 \\
431 \\
285 \\
67\end{array}$ & $\begin{array}{r}84 \\
362 \\
259 \\
53\end{array}$ & $\begin{array}{l}214 \\
148 \\
374 \\
126\end{array}$ & $\begin{array}{l}213 \\
172 \\
287 \\
159\end{array}$ & $\begin{array}{l}214 \\
148 \\
287 \\
159\end{array}$ & $\begin{array}{l}213 \\
172 \\
374 \\
126\end{array}$ \\
\hline & & & 522 & 476 & & & 882 & 758 & & & 808 & 885 \\
\hline
\end{tabular}


TABLE III-Continued

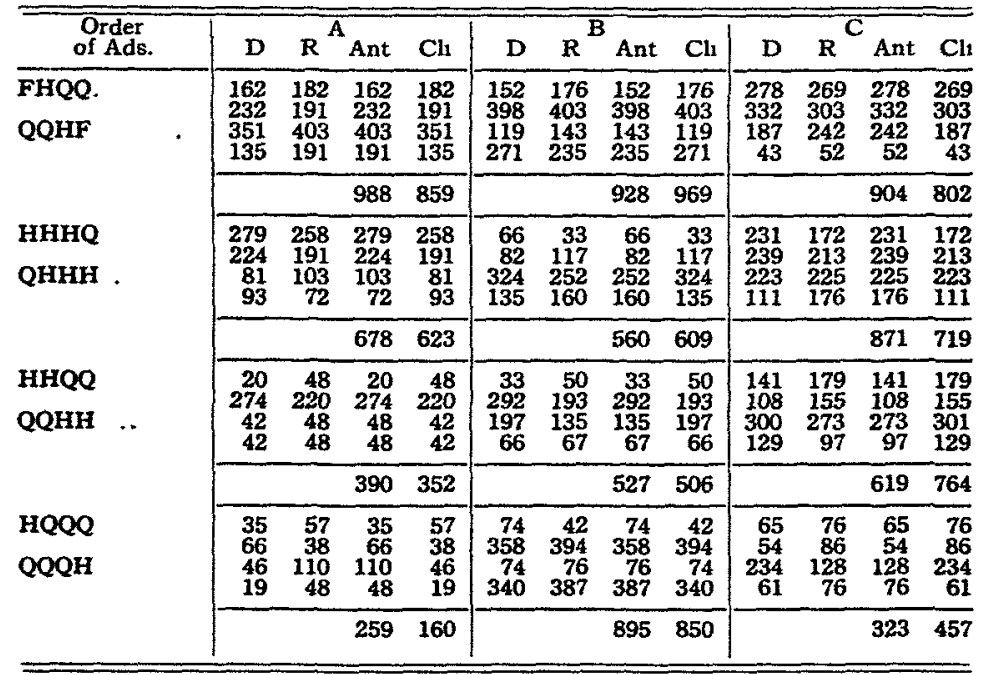

by 100 to avoid the use of decimal points. This data is presented complete in Table III, and in summarized form in Table IV. Again we have a suggestion that the anti-climax order is somewhat more efficient than in the climax.

TABLE IV

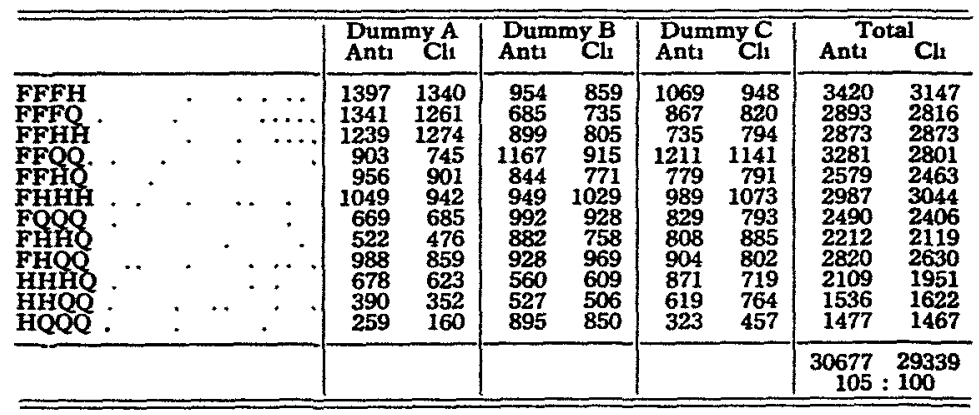

But again a difficulty of interpretation creeps in and this time it comes from the sheer magnitude of the figures themselves. The difference between 66 and 33 for instance is 33 or $100 \%$; the difference between 540 and 507 is again 33 but only about $6 \%$. There is to be found in the figures a dif- 
TABLE V

\begin{tabular}{|c|c|c|c|c|c|c|}
\hline & A & B & C & Total & Sum & Av. \\
\hline FFFH.......... & $\begin{array}{r}1071 \\
988 \\
1155 \\
942\end{array}$ & $\begin{array}{r}895 \\
1131 \\
1253 \\
1201\end{array}$ & $\begin{array}{r}1268 \\
973 \\
947 \\
1281\end{array}$ & $\begin{array}{l}3234 \\
3092 \\
3355 \\
3424\end{array}$ & 1300 & 2076 \\
\hline FFFQ $\ldots \ldots \ldots$ & $\begin{array}{r}1068 \\
1188 \\
1025 \\
895\end{array}$ & $\begin{array}{r}928 \\
1032 \\
621 \\
1079\end{array}$ & $\begin{array}{r}982 \\
1241 \\
1092 \\
910\end{array}$ & $\begin{array}{l}2978 \\
3461 \\
2738 \\
2884\end{array}$ & (20) & Jaro \\
\hline FFHH . & $\begin{array}{r}1060 \\
930 \\
899 \\
1022\end{array}$ & $\begin{array}{r}978 \\
1113 \\
1232 \\
980\end{array}$ & $\begin{array}{r}850 \\
504 \\
1621 \\
983\end{array}$ & $\begin{array}{r}2888 \\
2547 \\
3752 \\
2985\end{array}$ & 12061 & 3015 \\
\hline FFQQ $\ldots . . .$. & $\begin{array}{r}874 \\
1072 \\
1800 \\
1469\end{array}$ & $\begin{array}{r}1866 \\
965 \\
1370 \\
1249\end{array}$ & $\begin{array}{r}897 \\
1228 \\
1012 \\
1292\end{array}$ & $\begin{array}{l}3637 \\
3265 \\
4182 \\
4010\end{array}$ & 12172 & 3043 \\
\hline FFHQ.. & $\begin{array}{r}1221 \\
1028 \\
1000 \\
967\end{array}$ & $\begin{array}{r}2528 \\
1034 \\
1023 \\
941\end{array}$ & $\begin{array}{r}1040 \\
664 \\
1058 \\
1165\end{array}$ & $\begin{array}{l}4789 \\
2726 \\
3081 \\
3073\end{array}$ & 15094 & 3774 \\
\hline FHНH... & $\begin{array}{r}1171 \\
1460 \\
778 \\
1301\end{array}$ & $\begin{array}{r}1114 \\
822 \\
798 \\
1004\end{array}$ & $\begin{array}{r}700 \\
1121 \\
827 \\
837\end{array}$ & $\begin{array}{l}2985 \\
3403 \\
2403 \\
3142\end{array}$ & 13669 & 3417 \\
\hline FQQQ... & $\begin{array}{r}832 \\
1939 \\
1127 \\
892\end{array}$ & $\begin{array}{r}1069 \\
1164 \\
1191 \\
827\end{array}$ & $\begin{array}{r}1125 \\
1020 \\
1419 \\
873\end{array}$ & $\begin{array}{l}3026 \\
4123 \\
3737 \\
2592\end{array}$ & 11933 & \\
\hline FHHQ. & $\begin{array}{r}823 \\
1032 \\
1213 \\
1655\end{array}$ & $\begin{array}{l}1180 \\
1190 \\
1100 \\
1265\end{array}$ & $\begin{array}{r}1005 \\
861 \\
767 \\
1261\end{array}$ & $\begin{array}{l}8008 \\
3083 \\
3080 \\
4181\end{array}$ & 3478 & 3570 \\
\hline FHQQ $\ldots \ldots \ldots \ldots$ & $\begin{array}{r}889 \\
1214 \\
1150 \\
1413\end{array}$ & $\begin{array}{r}865 \\
987 \\
1200 \\
866\end{array}$ & $\begin{array}{l}1031 \\
1095 \\
1292 \\
1210\end{array}$ & $\begin{array}{l}2785 \\
3296 \\
3642 \\
3489\end{array}$ & & \\
\hline HHHQ.... & $\begin{array}{l}1080 \\
1172 \\
1274 \\
3574\end{array}$ & $\begin{array}{r}2000 \\
700 \\
778 \\
1186\end{array}$ & $\begin{array}{l}1342 \\
1122 \\
1010 \\
1586\end{array}$ & $\begin{array}{l}4422 \\
2994 \\
3062 \\
3546\end{array}$ & 1024 & 3506 \\
\hline
\end{tabular}


TABLE V-Contınued

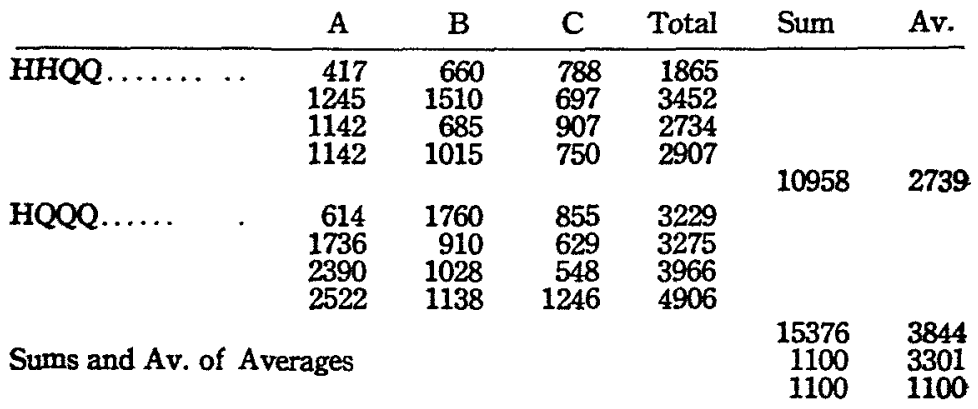

ference between the absolute amount and the relative amount of influence resulting from the changing order of presentation.

Table of Ratios. The climax order is given an arbitrary value of 1,000 and the anti-climax a relative value. Since the climax order has always the value 1,000 , it is omitted from the table. Table $\mathrm{V}$ was prepared, in which the anti-climax values are given in ratios, the climax order of presentation

\section{TABLE VI}

Anti-climax ratios put in the form of a frequency table.

\begin{tabular}{cr}
$\mathbf{V}$ & \multicolumn{1}{c}{$\mathbf{F}$} \\
\hline $40-59$ & 3 \\
$60-79$ & 15 \\
$80-99$ & 37 \\
$100-119$ & 49 \\
$120-139$ & 23 \\
$140-159$ & 6 \\
$160-179$ & 4 \\
$180-199$ & 3 \\
$200-219$ & 1 \\
$220-239$ & 1 \\
$240-259$ & 2
\end{tabular}

Av. $=11111$

A. D. $=2267$

$\mathrm{n}=144$

P. E. $=16-$

having an arbitrary value of 1,000 . This table in summary indicates that the anti-climax order is $10 \%$ more effective than the climax order, and this fact is emphasized by throwing the anti-climax values into a table of frequency as is done in Table VI. This method of presenting the data gives the anti-climax order a value of $11+\%$ advantage over the climax. The probable error is very low, only 16 , approxi- 
mately 1-7 of the significant difference. It may be concluded then, that the anti-climax order is more effective than the climax order.

GRAPH DRAWN FROM TABLE VI

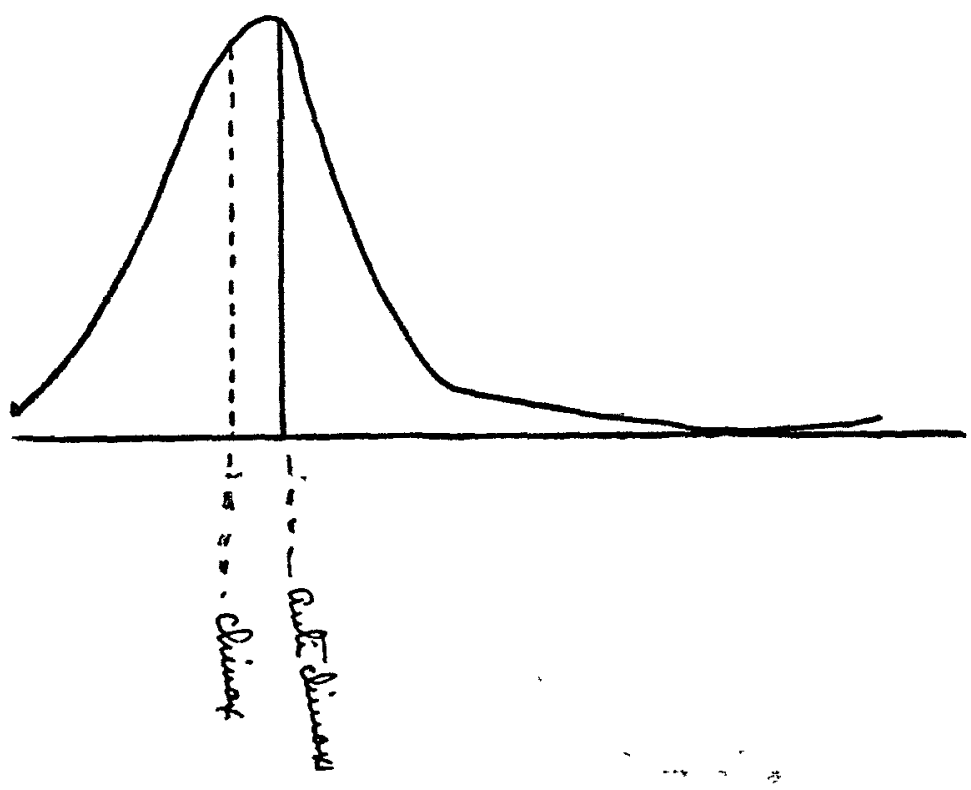

